\title{
Serum amyloid A, ferritin and carcinoembryonic antigen as biomarkers of severity in patients with COVID-19
}

\author{
DINA A. ABDELHAKAM ${ }^{1}$, FATMA MOHAMMED BADR ${ }^{2}$, MOHAMMED ABD EL MONEM TEAMA $^{2}$, \\ NOURAN M. BAHIG ELMIHI ${ }^{1}$ and MARWA ADHAM EL-MOHAMDY ${ }^{1}$ \\ Departments of ${ }^{1}$ Clinical Pathology and ${ }^{2}$ Internal Medicine and Rheumatology, \\ Faculty of Medicine, Ain Shams University, Cairo 11566, Egypt
}

Received August 18, 2021; Accepted November 18, 2021

DOI: $10.3892 /$ br.2021.1496

\begin{abstract}
In view of the rapid spread of COVID-19 and the high mortality rate of severe cases, reliable risk stratifying indicators of prognosis are necessary to decrease morbidity and mortality. The aim of the present study was to evaluate the value of serum amyloid A (SAA) and carcinoembryonic antigen (CEA) as prognostic biomarkers in comparison to other predictors, including C-reactive protein (CRP) and ferritin levels. This study included 124 patients diagnosed with COVID-19, and they were assigned to one of two groups: Mild and severe, based on the severity of the infection. Radiological and laboratory investigations were performed, including evaluation of CRP, ferritin, D-Dimer, SAA and CEA levels. Significantly higher levels of CRP, ferritin, D-Dimer, SAA and CEA were observed in severe cases. SAA was significantly correlated with CRP $(r=0.422$, $\mathrm{P}<0.001)$, ferritin $(\mathrm{r}=0.574, \mathrm{P}<0.001), \mathrm{CEA}(\mathrm{r}=0.514, \mathrm{P}<0.001)$ and computed tomography severity score (CT-SS; $\mathrm{r}=0.691$, $\mathrm{P}<0.001)$. CEA was correlated with CRP $(\mathrm{r}=0.441, \mathrm{P}<0.001)$, ferritin $(\mathrm{r}=0.349, \mathrm{P}<0.001)$ and CT-SS $(\mathrm{r}=0.374, \mathrm{P}<0.001)$. Receiver operator characteristic (ROC) curves for performance of SAA, CEA, ferritin, CRP and SAA showed the highest AUC
\end{abstract}

Correspondence to: Dr Dina A. Abdelhakam, Department of Clinical Pathology, Faculty of Medicine, Ain Shams University, 38 Abbassyia Square, Cairo 11566, Egypt

E-mail: dinaam@med.asu.edu.eg

Abbreviations: ARDS, acute respiratory distress syndrome; ALT, alanine transaminase; AUC, area under curve; AST, aspartate transaminase; BUN, blood urea nitrogen; CRP, C- reactive protein; CBC, complete blood count; CT, computed tomography; CO-RADS, COVID-19 Reporting and Data System; COVID-19, Coronavirus disease-2019; SARS-CoV-2, severe acute respiratory Coronavirus-2; CT-SS, CT severity score; IL, interleukin; IQR, interquartile range; ILD, interstitial lung diseases; CEA, carcinoembryonic antigen; NPV, negative predictive value; PLT, platelet; PPV, positive predictive value; ROC, receiver operating characteristic; RT-qPCR, reverse transcription-quantitative PCR; SAA, serum amyloid A; WBC, white blood count.

Key words: serum amyloid A, carcinoembryonic antigen, CT severity score, coronavirus, ferritin value of 0.928 , with a specificity of $93.1 \%$, and a sensitivity of $98.5 \%$ at a cut-off of $16 \mathrm{mg} / \mathrm{l}$. The multi-ROC curve for SAA and ferritin showed $100 \%$ specificity, $100 \%$ sensitivity and $100 \%$ efficiency, with an AUC of 1.000 . Thus, combining SAA and ferritin may have guiding significance for predicting COVID-19 severity. SAA alone showed the highest prognostic significance. Both SAA and CEA were positively correlated with the CT-SS. Early monitoring of these laboratory markers may thus provide significant input for halting disease progression and reducing mortality rates.

\section{Introduction}

Coronavirus disease 2019 (COVID-19) is caused by severe acute respiratory coronavirus 2 (SARS-CoV-2) (1). SARS-CoV-2 has led to a serious pandemic worldwide and has become a burden borne by health care systems since its first outbreak in Wuhan, China, in 2019 (2).

With the rapid spread of COVID-19 worldwide, researchers are working on the development of vaccines and effective therapeutics; however, the number of new cases and deaths continues to increase at unfavorable rates $(3,4)$. In view of the high mortality rates of severe cases, rapid reliable risk stratification tools and sensitive indicators of prognosis are urgently required for timely disease monitoring and efficient interventions targeting the reduction of morbidity and mortality (5).

The initial clinical picture of several patients with COVID-19 appears to be non-specific. Patients may present with minimal symptoms and no radiological abnormalities. In certain cases, rapid disease progression may occur, leading to acute respiratory distress syndrome (ARDS), multiple organ failure and even death (4,5). A cytokine storm has been identified in several studies as a key factor causing COVID-19 exacerbation or mortality (6). Several inflammatory factors, coagulation parameters and cytokines have been proposed as potential biomarkers of disease progression, occurrence of cytokine storm and severity (7).

Serum amyloid A (SAA) is an important potential biomarker. SAA is a highly conserved acute-phase protein primarily produced by the liver in response to inflammatory cytokines such as IL-6, IL-1, TNF, IFN- $\gamma$ and TGF- $\beta$. SAA levels are associated with the severity of inflammation (8). In addition, SAA is the precursor of the amyloid A protein, which is a fibrillar, insoluble product that is deposited in major 
organs, thus contributing to organ failure and death during the course of secondary amyloidosis (9).

Another important potential biomarker is carcinoembryonic antigen (CEA); a glycoprotein formed in the respiratory and colonic epithelium during embryogenesis, and it has been widely utilized as a tumor marker to monitor tumor progression (10). CEA is related to respiratory or digestive cancers and infectious diseases, such as gonorrhea, or chronic inflammatory diseases such as interstitial lung diseases (ILD) (11). Immunohistochemical staining of lung specimens from patients with pulmonary fibrosis demonstrated strong expression of CEA in the metaplastic bronchiolar and type II alveolar epithelia (12). Significant hyperplasia of type II alveolar epithelial cells and interstitial fibrosis have been described in several reports of COVID-19 autopsies and biopsies, similar to the pathological changes observed in ILD (13).

In the present study, SAA and CEA were evaluated as potential prognostic biomarkers in comparison to other commonly used inflammatory predictors, including C-reactive protein (CRP) and ferritin, and their association with the severity of COVID-19 and CT scan findings, and whether they may be a beneficial tool for patient stratification was assessed.

\section{Materials and methods}

Patients. This cross-sectional study included 124 patients diagnosed with COVID-19 enrolled from the Ain Shams University Isolation Hospital (Cairo, Egypt). In this study cohort, the median age was 48 years and the 25-75th IQR was 40-56 years (age range, $25-87$ years). A total of 10 patients were $>65$ years old, constituting $8 \%$ of the entire cohort. Of the 124 cases, 98 (79\%) were men and 26 were women $(21 \%)$. The median male age was 48.5 years (IQR, 40-54) and the median female age was 44.5 years (IQR, 35-57). Data were collected from hospitalized patients between September 2020 and February 2021. Patients were diagnosed and categorized into mild and severe groups according to the World Health Organization interim guidelines (14).

A definite COVID-19 case was identified as a positive result using sequencing or reverse transcription-quantitative PCR (RT-qPCR) of nasopharyngeal swabs.

The patients were classified into 2 groups: i) Mild group, had clinical symptoms of fever, fatigue, cough, anorexia, malaise, muscle pain, sore throat, dyspnea, nasal congestion and/or a headache; and ii) Severe group, had respiratory distress, a respiratory rate of $\geq 30$ breaths/min at resting state, a mean oxygen saturation of $\leq 93 \%$ and an arterial blood oxygen partial pressure $(\mathrm{PaO} 2)$ /oxygen concentration $(\mathrm{FiO} 2)$ of $\leq 300 \mathrm{mmHg}$.

The exclusion criteria were as follows: i) Patients infected with other viruses or bacteria; ii) patients diagnosed with autoimmune disorders; iii) patients diagnosed with arthritic diseases and iv) patients with cancer and/or any chronic disease related to elevated CEA and/or SAA levels, such as chronic kidney disease (15).

Ethical considerations. This study was conducted in accordance with the principles outlined in the Declaration of Helsinki of the World Medical Association (16). The study was approved by the Institutional Ethics Committee of Ain Shams University. Informed consent was obtained from all enrolled participants after receiving an explanation of the study's aim and procedures.
Methods. The recruited cohort consisted of 124 patients diagnosed with COVID-19 at the Ain Shams University Hospital. A detailed history was obtained for all patients, with a particular emphasis on age, sex, duration of disease and clinical symptoms. Data from routine investigations were retrieved, including liver enzyme levels [alanine transaminase (ALT) and aspartate transaminase (AST)], kidney function tests [blood urea nitrogen (BUN), creatinine], inflammatory parameters (CRP, ferritin) and D-Dimer levels, in addition to complete blood count (CBC), hemoglobin, white blood count (WBC), neutrophil count, lymphocyte count and platelet (PLT) count (15).

Detection of viral RNA was performed using the CerTest ViasureVR SARS-CoV-2 RT-qPCR Detection kit (CerTest, Biotec) according to the manufacturer's instructions. The detection was performed in a one-step real-time reverse-transcription format, where the reverse transcription and subsequent amplification of a specific target sequence occurred in the same reaction well. The isolated RNA target was transcribed to generate cDNA using the included reverse transcriptase, followed by the amplification of a conserved region of the open reading frames (ORF) $1 \mathrm{ab}$ and $\mathrm{N}$ genes for SARS-CoV-2 using specific primers and a fluorescent-labelled probe, all of which were included in the kit. The assay has a $97.5 \%$ sensitivity and $>99.9 \%$ specificity. The average estimated limit of detection for SARS-CoV-2 was 18 copies $/ \mathrm{ml}$.

Blood samples from PCR-positive patients were obtained, left to clot completely, and centrifuged at 3,000 x g for $20 \mathrm{~min}$ at $4^{\circ} \mathrm{C}$, and stored at $-80^{\circ} \mathrm{C}$ until required. SAA level analysis was performed using the Invitrogen human SAA ELISA kit (cat. no. EHSAA1; Invitrogen; Thermo Fisher Scientific, Inc.). The detection limit of this assay is $0.004 \mathrm{mg} / \mathrm{l}$. CEA serum concentrations were assayed using a Cobas e411 immunoassay autoanalyzer (Roche Diagnostics $\mathrm{GmbH}$ ).

Computed tomography (CT). All patients underwent non-contrast-enhanced chest CT in the Radiology Department of Ain Shams University, which was performed by expert radiologists using a Siemens 16-channel scope (CTAWP92544; Siemens Healthineers). The following CT parameters were evaluated: COVID-19 Reporting and Data System (CO-RADS) score based on CT findings (17): i) The CO-RADS score represents the level of suspicion of COVID-19. CO-RADS 1, COVID-19 is highly doubtful, CT is normal, or findings representing a non-infectious disease; CO-RADS 2, low level of suspicion of COVID-19 infection, CT findings consistent with other infections; CO-RADS 3, COVID-19 infection is indeterminate and unsure whether $\mathrm{CT}$ abnormalities are caused by COVID-19; CO-RADS 4, high suspicion level, and most CT findings are not extremely typical; CO-RADS 5, high level of suspicion with typical CT findings. ii) Semi quantitative scoring system: A quantitative estimate of pulmonary involvement based on abnormalities in the areas involved. The CT-severity score (CT-SS) is based on the extent of lobar involvement. Each of the five lung lobes was visually scored from $0-5$ as follows: 0 , no involvement; $1,<5 \%$ involvement; 2, 5-25\% involvement; $3,26-49 \%$ involvement; $4,50-75 \%$ involvement; and $5, \geq 75 \%$ involvement. The total CT score was the sum of individual lobar scores and ranged from 0 (no involvement) to 25 (maximum) (18). 
Table I. Demographics and baseline clinical, laboratory and radiological parameters of the COVID-19 patients.

\begin{tabular}{|c|c|c|c|c|}
\hline \multirow[b]{2}{*}{ Parameters } & \multirow[b]{2}{*}{ Value } & \multirow[b]{2}{*}{ Range } & \multicolumn{2}{|c|}{ Interquartile range } \\
\hline & & & 25th percentile & 75th percentile \\
\hline Age, years & 48 & $25-87$ & 40 & 56 \\
\hline Sex, n $(\%)$ & & - & - & - \\
\hline Male & $98(79)$ & & & \\
\hline Female & $26(21)$ & & & \\
\hline \multicolumn{5}{|l|}{ Clinical parameters } \\
\hline Duration of disease, days & 7 & $1-14$ & 4 & 10 \\
\hline Fever, n (\%) & $118(95.2)$ & - & - & - \\
\hline Cough, n (\%) & $108(87.1)$ & - & - & - \\
\hline Loss of smell and taste, $\mathrm{n}(\%)$ & $14(11.3)$ & - & - & - \\
\hline Dyspnea, $\mathrm{n}(\%)$ & $74(59.7)$ & - & - & - \\
\hline Respiratory distress, n (\%) & $39(31.5)$ & - & - & - \\
\hline Diarrhea, n (\%) & $8(6.6)$ & - & - & - \\
\hline \multicolumn{5}{|l|}{ Laboratory parameters } \\
\hline White blood cell count, 109/1 & 6.9 & $2.6-17$ & 4.3 & 10.3 \\
\hline Neutrophil count, 109/1 & 4.5 & $1.5-13.6$ & 2.82 & 7.2 \\
\hline Lymphocyte count, 109/1 & 1.22 & $0.46-4.38$ & 0.841 & 1.6 \\
\hline Platelet count, 109/1 & 232 & $50-649$ & 191 & 295 \\
\hline Hemoglobin, g/dl & 14.2 & $8.0-17.6$ & 13 & 15.1 \\
\hline Blood urea nitrogen, $\mathrm{mg} / \mathrm{dl}$ & 14 & $7.5-46$ & 11.7 & 19.2 \\
\hline Creatinine, $\mathrm{mg} / \mathrm{dl}$ & 0.8 & $0.47-7.5$ & 0.7 & 1.04 \\
\hline Aspartate transaminase, $\mathrm{U} / \mathrm{l}$ & 26 & $12-116$ & 17 & 43 \\
\hline Alanine transaminase, $\mathrm{U} / \mathrm{l}$ & 32 & $11-346$ & 16 & 54 \\
\hline C-reactive protein, mg/l & 43 & $1.8-444.0$ & 18 & 161.3 \\
\hline Ferritin, ng/ml & 361 & $24-2,450$ & 203.75 & 839.75 \\
\hline D-Dimer, ng/ml & 500 & $90-6088$ & 300 & 1200 \\
\hline Serum amyloid $\mathrm{A}, \mathrm{mg} / \mathrm{l}$ & 22.5 & $3-90$ & 10 & 49 \\
\hline Carcinoembryonic antigen, $\mathrm{ng} / \mathrm{ml}$ & 6 & $3-18$ & 5 & 9 \\
\hline \multicolumn{5}{|l|}{ Radiological parameters } \\
\hline CO-RADS score & 3 & $0-5$ & 1 & 4 \\
\hline Computed tomography-severity score & 8.5 & $0-25$ & 3 & 16 \\
\hline
\end{tabular}

CO-RADS, COVID-19 Reporting and Data System.

Statistical analysis. Statistical analysis was performed using SPSS version 25.0 (IBM Corp.). Continuous variables are presented as the median and interquartile range (IQR), whereas categorical variables are presented as the number (n) and percentage $(\%)$ of patients. A Wilcoxon rank sum test was used to evaluate differences between groups. A $\chi^{2}$ was used for analysis of sets of categorical data, and a Spearman's rank correlation test was used to measure the degree of correlation between the hierarchically ordered variables in this study. A multivariate regression analysis was performed. The selection of independent variables as potential predictors of COVID-19 severity and CT-SS included age, duration of disease, CBC (WBC, neutrophil, lymphocyte, PLT, monocyte, eosinophil count and hemoglobin), creatinine, AST, ALT, BUN, CRP, ferritin, D-Dimer, CEA and SAA. Stepwise multivariate-regression analysis was performed including for all studied items as independent variables (Model-1). The regression was re-run using only the most significant items with exclusion of the non-significant items iteratively. The least sensitive predictors for the model had the highest F-ratio and the lowest $\mathrm{P}$-value. $\mathrm{P}<0.05$ was considered to indicate a statistically significant difference. Receiver operating characteristic (ROC) curves were used for predictive analysis by calculating the area under the curve (AUC), sensitivity and specificity.

\section{Results}

A total of 124 patients whose RT-qPCR tests for COVID-19 were positive were recruited, their baseline demographics, clinical characteristics, laboratory, radiological CO-RADS score and CT-SS parameters are summarized in Table I, including age, duration of disease, symptoms, laboratory 
Table II. Comparison between mild and severe COVID-19 patients.

\begin{tabular}{|c|c|c|c|c|c|c|}
\hline Parameter & Mild, $\mathrm{n}=58$ & Severe, $n=66$ & $\mathrm{Z}$ & $\chi^{2}$ & P-value & Significance \\
\hline Age, years (IQR) & $43(35-52.5)$ & $50(43-56)$ & -3.24 & 2.88 & $<0.001$ & HS \\
\hline $\operatorname{Sex}, \mathrm{n}(\%)$ & & & & & $>0.05$ & NS \\
\hline Male & $42 / 58(72.4)$ & $56 / 66(84.8)$ & & & & \\
\hline Female & $16 / 58(27.6)$ & $10 / 66(15.2)$ & & & & \\
\hline \multicolumn{7}{|l|}{ Clinical parameters } \\
\hline Duration of disease, days (IQR) & $5(4-9)$ & $8(6-11)$ & -3.7 & & $<0.001$ & HS \\
\hline Fever, $\mathrm{n}(\%)$ & $52(89.7)$ & $66(100)$ & & 7.175 & $<0.05$ & $\mathrm{~S}$ \\
\hline Cough, n (\%) & $48(82.7)$ & $60(90.9)$ & & 1.825 & $>0.05$ & NS \\
\hline Loss of smell, n (\%) & $4(6.8)$ & $10(15.1)$ & & 2.1 & $>0.05$ & NS \\
\hline Dyspnea, n (\%) & $26(44.8)$ & $48(72.7)$ & & 9.986 & $<0.05$ & $\mathrm{~S}$ \\
\hline Respiratory distress, n (\%) & $1(1.7)$ & $38(57.6)$ & & 41.39 & $<0.001$ & HS \\
\hline Diarrhea, n (\%) & $4(6.8)$ & $4(6.1)$ & & 0.036 & $>0.05$ & NS \\
\hline \multicolumn{7}{|l|}{ Laboratory parameters } \\
\hline White blood cell count, 109/1, median (IQR) & $5.5(4.3-10.5)$ & $7.8(4.8-10.4)$ & -1.02 & & $>0.05$ & NS \\
\hline Neutrophil count, 109/1, median (IQR) & $4(2.7-6.9)$ & $5.5(2.9-8.3)$ & -1.66 & & $>0.05$ & NS \\
\hline Lymphocyte count, $10^{9} / 1$, median (IQR) & $1.24(0.9-1.7)$ & $1.1(0.8-1.5)$ & -0.62 & & $>0.05$ & NS \\
\hline Platelet count, $10^{9} / 1$, median (IQR) & $229(194-259)$ & $246(168-330)$ & -0.62 & & $>0.05$ & NS \\
\hline Hemoglobin, g/dl, median (IQR) & $14.2(11.9-15.4)$ & $14(13.2-15)$ & -0.58 & & $>0.05$ & NS \\
\hline Blood urea nitrogen, mg/dl, median (IQR) & $14(11.5-16.9)$ & $15.9(11.7-22)$ & -2.16 & & $<0.05$ & $\mathrm{~S}$ \\
\hline Creatinine, mg/dl, median (IQR) & $0.8(0.7-1.03)$ & $0.9(0.7-1.05)$ & -0.54 & & $>0.05$ & NS \\
\hline Aspartate transaminase, $\mathrm{U} / \mathrm{l}$, median (IQR) & $23(15.5-37.25)$ & $34(18-43.25)$ & -1.85 & & $>0.05$ & NS \\
\hline Alanine transaminase, $\mathrm{U} / \mathrm{l}$, median (IQR) & $27(15-51)$ & $34(17-54)$ & -0.96 & & $>0.05$ & NS \\
\hline C-reactive protein, mg/l, median (IQR) & $22(11.8-48.5)$ & $83(29-222)$ & -4.51 & & $<0.001$ & HS \\
\hline Ferritin, ng/ml, median (IQR) & $227(127-290)$ & $778(455-1,225)$ & -7.86 & & $<0.001$ & HS \\
\hline D-Dimer, ng/ml, median (IQR) & $336(230-451)$ & $1200(569-1,451)$ & -7.09 & & $<0.001$ & HS \\
\hline Serum amyloid A, mg/l, median (IQR) & $10(7-12)$ & $48.5(34-59)$ & -9.28 & & $<0.001$ & HS \\
\hline Carcinoembryonic antigen, $\mathrm{ng} / \mathrm{ml}$, median (IQR) & $5(5-7)$ & $9(6-13)$ & -6.04 & & $<0.001$ & HS \\
\hline \multicolumn{7}{|l|}{ Radiological parameters } \\
\hline CO-RADS score, median (IQR) & $1(0.2)$ & $4(3-5)$ & -8.1 & & $<0.001$ & HS \\
\hline Computed tomography-severity score & $3(2-8)$ & $15(8.7-20)$ & -6.82 & & $<0.001$ & HS \\
\hline
\end{tabular}

S, significant; HS, highly significant; NS, not significant; IQR, inter-quartile range; CO-RADS, COVID-19 Reporting and Data System.

parameters including CBC, liver enzymes (AST and ALT), kidney function tests (BUN and creatinine), inflammatory parameters (CRP, ferritin, and D-Dimer), in addition to SAA and CEA levels. In this study cohort, the median age was 48 years and the $25-75$ th IQR was $40-56$ years (age range, $25-87$ years). A total of 10 patients were $>65$ years old, constituting $8 \%$ of the entire cohort. Of the 124 cases, 98 (79\%) were men and 26 were women $(21 \%)$. The median male age was 48.5 years (IQR, 40-54) and the median female age was 44.5 years (IQR, 35-57) $(\mathrm{P}>0.05)$.

All patients were divided into two groups as shown in Table II: Mild or severe COVID-19 infection. Of the 124 patients, 58 patients $(46.7 \%)$ were classified as having mild COVID-19, with a median age of 43 years (IQR, 35-52.5) and 66 patients $(53.3 \%)$ were classified as having severe COVID-19, with a median age of 50 years (IQR, 43-56); this showed a highly significant statistical difference between both groups with median ages higher in the severe group $(\mathrm{P}<0.001)$.
In the severe COVID-19 group, 7 out of $66(10.6 \%)$ patients were $>65$ years old compared to three cases out of $58(5.2 \%)$ in the mild group.

All studied parameters were compared in both groups and are presented in Table II. Patients with severe COVID-19 $(n=66)$ showed a statistically significant higher median disease duration of 8 days (IQR, 6-11), while the remaining 58 mild cases had a median duration of disease of 5 days (IQR, 4-9) $(\mathrm{P}<0.001)$. Regarding laboratory parameters, such as total WBC, neutrophil, lymphocyte counts, PLT, creatinine, ALT and AST, there were no significant differences between the groups ( $\mathrm{P}>0.05$; Table II). In the entire cohort, it was noticed that high serum levels of inflammatory parameters, including CRP, ferritin and D-Dimer, as well as SAA and CEA, were predictors of disease severity (Table I); their levels in severe COVID-19 group were significantly higher than that in the mild group $(\mathrm{P}<0.001)$. Patients with mild COVID-19 showed median SAA levels of $10 \mathrm{ng} / \mathrm{ml}$ (IQR, 7-12), while 
Table III. Correlation between SAA and CEA concentration with all the studied parameters.

\begin{tabular}{|c|c|c|c|c|c|c|}
\hline \multirow[b]{2}{*}{ Parameter } & \multicolumn{3}{|c|}{ SAA } & \multicolumn{3}{|c|}{ CEA } \\
\hline & $\mathrm{R}$ & P-value & Significance & $\mathrm{R}$ & P-value & Significance \\
\hline Age & 0.349 & $<0.001$ & HS & 0.328 & $<0.001$ & HS \\
\hline Duration of disease & 0.337 & $<0.001$ & HS & 0.212 & $<0.05$ & $\mathrm{~S}$ \\
\hline White blood cell count & 0.12 & $>0.05$ & NS & -0.026 & $>0.05$ & NS \\
\hline Neutrophil count & 0.179 & $<0.05$ & $\mathrm{~S}$ & 0.072 & $>0.05$ & NS \\
\hline Lymphocyte count & -0.06 & $>0.05$ & NS & -0.155 & $>0.05$ & NS \\
\hline Platelet count & 0.07 & $>0.05$ & NS & 0.007 & $>0.05$ & NS \\
\hline Hemoglobin & -0.011 & $>0.05$ & NS & 0.023 & $>0.05$ & NS \\
\hline Blood urea nitrogen & 0.345 & $<0.001$ & HS & 0.296 & $<0.001$ & HS \\
\hline Creatinine & 0.118 & $>0.05$ & NS & 0.15 & $>0.05$ & NS \\
\hline Aspartate transaminase & 0.149 & $>0.05$ & NS & 0.149 & $>0.05$ & NS \\
\hline Alanine transaminase & 0.092 & $>0.05$ & NS & 0.052 & $>0.05$ & NS \\
\hline C- reactive protein & 0.422 & $<0.001$ & HS & 0.441 & $<0.001$ & HS \\
\hline Ferritin & 0.574 & $<0.001$ & HS & 0.349 & $<0.001$ & HS \\
\hline D-Dimer & 0.613 & $<0.001$ & HS & 0.309 & $<0.001$ & HS \\
\hline Carcinoembryonic antigen & 0.514 & $<0.001$ & HS & & & \\
\hline CO-RADS score & 0.812 & $<0.001$ & HS & 0.434 & $<0.001$ & HS \\
\hline Computed tomography severity score & 0.691 & $<0.001$ & HS & 0.374 & $<0.001$ & HS \\
\hline
\end{tabular}

S, significant; HS, highly significant; NS, not significant; IQR, inter-quartile range; CO-RADS, COVID-19 Reporting and Data System; SAA, serum amyloid A; CEA, carcinoembryonic antigen.

in the severe COVID-19 group, the median SAA was 48.5 (IQR, 34-59), which was significantly higher than that in the mild group $(\mathrm{P}<0.001)$. The median CEA level was $5 \mathrm{ng} / \mathrm{ml}$ (IQR,5-7) while in the severe group, the median CEA level was 9 (IQR, 6-13), which was significantly higher than that in the mild group $(\mathrm{P}<0.001)$ Table II. The median CT-SS was significantly higher in the severe group (median, 15; IQR, 8.75-20) than in the mild COVID-19 group (median, 3; IQR, 2-8) $(\mathrm{P}<0.001)$.

Using Spearman's rank correlation analysis, SAA showed a highly significant positive correlation with age $(r=0.349$, $\mathrm{P}<0.001)$ and disease duration $(\mathrm{r}=0.337, \mathrm{P}<0.001)$. SAA showed a significant positive correlation with neutrophil count $(\mathrm{r}=0.179, \mathrm{P}<0.05)$ and a highly significant positive correlation with all inflammatory indices, including CRP $(r=0.422, \mathrm{P}<0.001)$, ferritin $(\mathrm{r}=0.574, \mathrm{P}<0.001)$, D-Dimer $(\mathrm{r}=0.613, \mathrm{P}<0.001)$ and CEA $(\mathrm{r}=0.514, \mathrm{P}<0.001)$. In addition, SAA showed a highly significant positive correlation with the CO-RADS score $(\mathrm{r}=0.812, \mathrm{P}<0.001)$ and CT-SS $(\mathrm{r}=0.691$, $\mathrm{P}<0.001$ ) (Table III).

CEA showed a highly significant positive correlation with age $(r=0.328, P<0.001)$, a significant positive correlation with disease duration $(\mathrm{r}=0.212, \mathrm{P}<0.05)$ and a highly significant positive correlation with all inflammatory indices, including CRP $(r=0.441, \mathrm{P}<0.001)$, ferritin $(\mathrm{r}=0.349, \mathrm{P}<0.001)$ and $\mathrm{D}$-Dimer $(\mathrm{r}=0.309, \mathrm{P}<0.001)$. CEA showed a highly significant positive correlation with the CO-RADS score $(r=0.434$, $\mathrm{P}<0.001)$ and CT-SS $(\mathrm{r}=0.374, \mathrm{P}<0.001)$ (Table III).

In the multivariate regression model in Table IV, including age, disease duration, CBC, SAA, ferritin, CEA, CRP,
D-Dimer, BUN, creatinine, AST and ALT, both SAA and ferritin were the best independent predictors of COVID-19 severity $(\mathrm{F}=170.861, \mathrm{P}<0.001)$. Furthermore, in another multivariate regression model for predictors of CT-SS and pulmonary involvement (Table V), SAA and PLT count were independent predictors of CT-SS and lung involvement $(\mathrm{F}=58.014, \mathrm{P}<0.001)$.

ROC curves showing the performance of SAA, CEA, ferritin and CRP levels in predicting severity in COVID-19 patients, cutoff value and performance characteristics are presented in Table VI and Fig. 1. An SAA value of 16 was predictive of a poor prognosis with a specificity of $93.1 \%$, sensitivity of 98.5 , positive predictive value (PPV) of 94.2, negative predictive value (NPV) of 98.2, efficiency of 96, and an AUC of 0.928. In a multi-ROC curve showing the performance of SAA in addition to ferritin. Specificity, sensitivity, PPV, NPV and efficiency were $100 \%$ and AUC was equal to 1.000 .

\section{Discussion}

COVID-19 is an acute infectious disease caused by SARS-CoV-2. The initial clinical picture of several patients may be non-specific. Patients may present with minimal symptoms, including mild to moderate fever, chills, malaise, respiratory distress and/or gastroenterological disorders such as nausea and vomiting (19). In several cases, rapid disease progression can occur within a few days with ARDS, uncompensated acidosis, septic shock and coagulation dysfunction, followed by multiple organ failure and death $(4,5,20)$. 
Table IV. Regression model outcomes of significant predictors of COVID-19 severity.

A, Model 1

\begin{tabular}{|c|c|c|c|c|c|c|c|}
\hline Item & Regression coefficient & $\mathrm{t}$ & P-value & Significance & F-Ratio & P-value & Significance \\
\hline Constant & 0.555 & 1.996 & $<0.05$ & $\mathrm{~S}$ & & & \\
\hline Age, years & 0.001 & 0.527 & $>0.05$ & NS & & & \\
\hline Duration of disease, days & 0.004 & 0.496 & $>0.05$ & NS & & & \\
\hline White blood cell count, $10^{9} / 1$ & -0.12 & -1.18 & $>0.05$ & NS & & & \\
\hline Neutrophil count, $10^{9} / 1$ & 0.123 & 1.238 & $>0.05$ & NS & & & \\
\hline Lymphocyte count, $10^{9} / 1$ & 0.164 & 1.557 & $>0.05$ & NS & & & \\
\hline Platelet count, $10^{9} / 1$ & 0 & 0.446 & $>0.05$ & NS & & & \\
\hline C-reactive protein, $\mathrm{mg} / \mathrm{l}$ & -0.0000972 & -0.284 & $>0.05$ & NS & & & \\
\hline Ferritin, ng/ml & 0 & 4.36 & $<0.001$ & HS & & & \\
\hline D-Dimer, ng/ml & $5.795 \mathrm{E}-05$ & 1.795 & $>0.05$ & NS & & & \\
\hline Carcinoembryonic antigen, $\mathrm{ng} / \mathrm{ml}$ & 0.026 & 3.045 & $<0.05$ & $\mathrm{~S}$ & & & \\
\hline Serum amyloid A, mg/l & 0.01 & 5.297 & $<0.001$ & HS & & & \\
\hline Monocyte count, $10^{9} / 1$ & 0.07 & 0.522 & $>0.05$ & NS & & & \\
\hline Eosinophil count, 109/1 & 0.04 & 0.236 & $>0.05$ & NS & & & \\
\hline Hemoglobin, g/dl & 0.017 & 1.283 & $>0.05$ & NS & & & \\
\hline Blood urea nitrogen, mg/dl & -0.007 & -1.742 & $>0.05$ & NS & & & \\
\hline Creatinine, $\mathrm{mg} / \mathrm{dl}$ & -0.07 & -1.828 & $>0.05$ & NS & & & \\
\hline Aspartate transaminase, $\mathrm{U} / \mathrm{l}$ & 0.003 & 1.688 & $>0.05$ & NS & & & \\
\hline Alanine transaminase, $\mathrm{U} / \mathrm{l}$ & -0.002 & -2.606 & $<0.05$ & $\mathrm{~S}$ & & & \\
\hline CO-RADS score & 0.043 & 1.218 & $>0.05$ & NS & & & \\
\hline CT Severity score & -0.001 & -0.18 & $>0.05$ & NS & & & \\
\hline Overall & & & & & 22.574 & $<0.001$ & HS \\
\hline
\end{tabular}

B, Model 2

\begin{tabular}{|c|c|c|c|c|c|c|c|}
\hline Item & Regression coefficient & $\mathrm{t}$ & P-value & Significance & F-Ratio & P-value & Significance \\
\hline Constant & 0.854 & 14.114 & $<0.001$ & HS & & & \\
\hline Ferritin, ng/ml & 0 & 6.903 & $<0.001$ & HS & & & \\
\hline D-Dimer, ng/ml & 0.0000732 & 2.723 & $<0.05$ & S & & & \\
\hline Carcinoembryonic antigen, $\mathrm{ng} / \mathrm{ml}$ & 0.027 & 3.577 & $<0.05$ & $\mathrm{~S}$ & & & \\
\hline Serum amyloid $\mathrm{A}, \mathrm{mg} / \mathrm{l}$ & 0.012 & 10.453 & $<0.001$ & HS & & & \\
\hline Blood urea nitrogen, mg/dl & -0.004 & -1.237 & $>0.05$ & NS & & & \\
\hline Creatinine, mg/dl & -0.105 & -3.245 & $<0.05$ & $\mathrm{~S}$ & & & \\
\hline Aspartate transaminase, $\mathrm{U} / \mathrm{l}$ & 0.003 & 2.317 & $<0.05$ & $\mathrm{~S}$ & & & \\
\hline Alanine transaminase, $\mathrm{U} / \mathrm{l}$ & -0.002 & -2.827 & $<0.05$ & $\mathrm{~S}$ & & & \\
\hline Overall & & & & & 60.933 & $<0.001$ & HS \\
\hline
\end{tabular}

C, Model 3

\begin{tabular}{|c|c|c|c|c|c|c|c|}
\hline Item & Regression coefficient & $\mathrm{t}$ & P-value & Significance & F-Ratio & P-value & Significance \\
\hline Constant & 0.871 & 16.09 & $<0.001$ & HS & & & \\
\hline Ferritin, ng/ml & 0 & 6.748 & $<0.001$ & HS & & & \\
\hline D-Dimer, ng/ml & $8.154 \mathrm{E}-05$ & 3.007 & $<0.05$ & $\mathrm{~S}$ & & & \\
\hline Carcinoembryonic antigen, $\mathrm{ng} / \mathrm{ml}$ & 0.026 & 3.398 & $<0.05$ & $\mathrm{~S}$ & & & \\
\hline Serum amyloid A, mg/l & 0.012 & 10.381 & $<0.001$ & HS & & & \\
\hline Creatinine, $\mathrm{mg} / \mathrm{dl}$ & -0.116 & -4.421 & $<0.001$ & HS & & & \\
\hline Alanine transaminase, $\mathrm{U} / \mathrm{l}$ & -0.001 & -1.91 & 0.059 & NS & & & \\
\hline Overall & & & & & 77.565 & $<0.001$ & HS \\
\hline
\end{tabular}


Table IV. Continued.

D, Model 4

\begin{tabular}{|c|c|c|c|c|c|c|c|}
\hline Item & Regression coefficient & $\mathrm{t}$ & P-value & Significance & F-Ratio & P-value & Significance \\
\hline Constant & 0.856 & 15.983 & $<0.001$ & HS & & & \\
\hline Ferritin, ng/ml & 0 & 6.979 & $<0.001$ & $\mathrm{HS}$ & & & \\
\hline Carcinoembryonic antigen, $\mathrm{ng} / \mathrm{ml}$ & 0.026 & 3.329 & $<0.05$ & $\mathrm{~S}$ & & & \\
\hline Serum amyloid $\mathrm{A}, \mathrm{mg} / \mathrm{l}$ & 0.013 & 11.272 & $<0.001$ & HS & & & \\
\hline Creatinine, $\mathrm{mg} / \mathrm{dl}$ & -0.116 & -4.263 & $<0.001$ & HS & & & \\
\hline Overall & & & & & 105.489 & $<0.001$ & HS \\
\hline
\end{tabular}

\section{E, Model 5}

\begin{tabular}{|c|c|c|c|c|c|c|c|}
\hline Item & Regression coefficient & $\mathrm{t}$ & P-value & Significance & F-Ratio & P-value & Significance \\
\hline Constant & 0.97 & 22.718 & $<0.001$ & HS & & & \\
\hline Ferritin, ng/ml & 0 & 6.923 & $<0.001$ & HS & & & \\
\hline Serum amyloid A, mg/l & 0.015 & 13.539 & $<0.001$ & HS & & & \\
\hline Creatinine, $\mathrm{mg} / \mathrm{dl}$ & -0.087 & -3.239 & $<0.05$ & $\mathrm{~S}$ & & & \\
\hline Overall & & & & & 126.339 & $<0.001$ & HS \\
\hline
\end{tabular}

F, Model 6

\begin{tabular}{|c|c|c|c|c|c|c|c|}
\hline Item & Regression coefficient & $\mathrm{t}$ & P-value & Significance & F-Ratio & $\mathrm{P}$-value & Significance \\
\hline Constant & 0.915 & 22.492 & $<0.001$ & HS & & & \\
\hline Ferritin, ng/ml & 0 & 6.007 & $<0.001$ & HS & & & \\
\hline Serum amyloid A, mg/l & 0.015 & 12.889 & $<0.001$ & HS & & & \\
\hline Overall & & & & & 170.861 & $<0.001$ & HS \\
\hline
\end{tabular}

S, significant; HS, highly significant; NS, not significant; CO-RADS, COVID-19 Reporting and Data System; Constant, the average value of response variable in the absence of all predictors.

The severity and prognosis of COVID-19 are complicated by the diversity of symptoms, imaging manifestations and the degree of disease progression $(21,22)$. Consequently, rapid reliable risk stratification tools and sensitive indicators are required for timely disease monitoring to develop efficient interventions targeting the reduction of morbidity and mortality in patients with COVID-19.

RT-qPCR is the primary technique used for accurate COVID-19 diagnosis. Although PCR detection can determine whether a patient is infected with COVID-19 (23), this technique cannot detect disease progression and severity. Several modalities are used to aid the diagnosis and provide evidence of disease progression, including chest radiography, CT scans and biomarkers.

Imaging methods, primarily CT scans, can identify chest infections and provide a reference for pathogen type determination. However, the risks related to patient transportation and examination, in addition to the extra protection required for healthcare personnel during the examination, can be a disadvantage $(21,24)$. Several studies have focused on the role of inflammatory markers as predictors of severity of COVID-19 infection, including lymphocyte and PLT counts, and CRP, D-Dimer and ferritin levels $(7,25,26)$.
Considering the above, the current study aimed to evaluate potential biomarkers, including SAA and CEA, in addition to other commonly used inflammatory predictors, such as CRP and ferritin, and their association with COVID-19 severity and CT scan findings, and to determine their role as beneficial tools for patient stratification.

Based on COVID-19 severity, the recruited cohort was divided into two groups: Patients with mild and patients with severe COVID-19. The median age of the mild group was 43 years, whereas that of the severe group was 50 years, and the difference was highly significant, suggesting an increased probability of severe disease in the elderly, as stated by several studies. These results agree with a study by Zheng et al (27), where the median age of 161 patients with COVID-19 admitted to Changsha Public Health Centre was 45 years, the median age of the severe group was 57 years, and the non-severe group was 40 years, showing a statistical difference in age between the two groups. Older age is a risk factor for a more severe infectious course, and several clinical studies on influenza virus pneumonia have shown that old age is a risk factor for severe illness, especially when associated with at least one underlying disease $(27,28)$. The results of the present study also 
Table V. Multivariate regression models of significant predictors of lung CT severity score.

A, Model 1

\begin{tabular}{|c|c|c|c|c|c|c|c|}
\hline Item & Regression coefficient & $\mathrm{t}$ & P-value & Significance & F-Ratio & P-value & Significance \\
\hline Constant & 2.865 & 0.448 & $>0.05$ & NS & & & \\
\hline Age, years & -0.035 & -0.647 & $>0.05$ & NS & & & \\
\hline Duration of disease, days & 0.245 & 1.387 & $>0.05$ & NS & & & \\
\hline White blood cell count, $10^{9} / 1$ & -1.92 & -0.832 & $>0.05$ & NS & & & \\
\hline Neutrophil count, $10^{9} / 1$ & 1.765 & 0.781 & $>0.05$ & NS & & & \\
\hline Lymphocyte count, $10^{9} / 1$ & 0.877 & 0.368 & $>0.05$ & NS & & & \\
\hline Platelet count, $10^{9} / 1$ & -0.013 & -2.12 & $<0.05$ & $\mathrm{~S}$ & & & \\
\hline C-reactive protein, mg/l & -0.005 & -0.686 & $>0.05$ & NS & & & \\
\hline Ferritin, ng/ml & 0.001 & 0.993 & $>0.05$ & NS & & & \\
\hline D-Dimer, ng/ml & 0.000 & 0.149 & $>0.05$ & NS & & & \\
\hline Carcinoembryonic antigen, $\mathrm{ng} / \mathrm{ml}$ & -0.097 & -0.488 & $>0.05$ & NS & & & \\
\hline Serum amyloid A, mg/l & 0.213 & 6.475 & $<0.001$ & HS & & & \\
\hline Monocyte count, $10^{9} / 1$ & 3.773 & 1.217 & $>0.05$ & NS & & & \\
\hline Eosinophil count, $10^{9} / 1$ & 0.101 & 0.027 & $>0.05$ & NS & & & \\
\hline Hemoglobin, g/dl & 0.316 & 1.009 & $>0.05$ & NS & & & \\
\hline Blood urea nitrogen, mg/dl & 0.214 & 2.29 & $<0.05$ & $\mathrm{~S}$ & & & \\
\hline Creatinine, $\mathrm{mg} / \mathrm{dl}$ & -1.302 & -1.479 & $>0.05$ & NS & & & \\
\hline Aspartate transaminase, $\mathrm{U} / 1$ & -0.007 & -0.182 & $>0.05$ & NS & & & \\
\hline Alanine transaminase, $\mathrm{U} / 1$ & 0.006 & 0.375 & $>0.05$ & NS & & & \\
\hline Overall & & & & & 7.991 & $<0.001$ & HS \\
\hline
\end{tabular}

B, Model 2

\begin{tabular}{|c|c|c|c|c|c|c|c|}
\hline Item & Regression coefficient & $\mathrm{t}$ & P-value & Significance & F-Ratio & P-value & Significance \\
\hline Constant & 5.446 & 3.519 & $<0.001$ & HS & & & \\
\hline Platelet count, $10^{9} / 1$ & -0.016 & -3.345 & $<0.001$ & HS & & & \\
\hline Serum amyloid A, mg/l & 0.219 & 9.552 & $<0.001$ & HS & & & \\
\hline Blood urea nitrogen, $\mathrm{mg} / \mathrm{dl}$ & 0.125 & 1.937 & $>0.05$ & NS & & & \\
\hline Overall & & & & & 40.805 & $<0.001$ & HS \\
\hline
\end{tabular}

C, Model 3

\begin{tabular}{|c|c|c|c|c|c|c|c|}
\hline Item & Regression coefficient & $\mathrm{t}$ & P-value & Significance & F-Ratio & P-value & Significance \\
\hline Constant & 6.817 & 4.898 & $<0.001$ & HS & & & \\
\hline Platelet count, $10^{9} / 1$ & -0.015 & -3.108 & $<0.001$ & HS & & & \\
\hline Serum amyloid A, mg/l & 0.234 & 10.642 & $<0.001$ & HS & & & \\
\hline Overall & & & & & 58.014 & $<0.001$ & HS \\
\hline
\end{tabular}

S, significant; HS, highly significant; NS, not significant; Constant, the average value of response variable in the absence of all predictors.

agreed with the results of Liu et al (29), in which it was stated that age was an important risk factor for severity in patients with COVID-19. The median age of the non-severe group was 43 years, and that of the severe group was 64 years $(27,29)$. In the current study, 10 patients were $>65$ years old, constituting $8 \%$ of the entire cohort. In the severe COVID-19 group, 7 patients out of $66(10.6 \%)$ compared to 3 cases out of $58(5.2 \%)$ in the mild group were $>65$ years taking into consideration that the current life expectancy for the Egyptian population in 2021 is 74.3 years, and the number of individuals aged $\geq 60$ years is 6.8 million, representing $6.7 \%$ of the total population according to official data by the Central Agency for Public Mobilization and Statistics (CAPMAS, 2021) (30).

A meta-analysis conducted by Peckham et al (31) on $3,111,714$ cases worldwide reported no difference in the proportion of confirmed COVID-19 cases between men and 
Table VI. Cutoff and performance characteristics of SAA, CEA, ferritin and CRP in predicting severity in COVID-19 patients.

\begin{tabular}{lcccccc}
\hline Parameter & Cutoff & $\begin{array}{c}\text { Sensitivity, } \\
\%\end{array}$ & $\begin{array}{c}\text { Specificity, } \\
\%\end{array}$ & $\begin{array}{c}\text { Positive } \\
\text { predictive value }\end{array}$ & $\begin{array}{c}\text { Negative } \\
\text { predictive value }\end{array}$ & $\begin{array}{c}\text { Area under } \\
\text { Efficiency } \\
\text { the curve }\end{array}$ \\
\hline SAA, mg/l & 16 & 98.5 & 93.1 & 94.2 & 98.2 & 96 \\
CEA, ng/ml & 7 & 63.6 & 86.2 & 84 & 67.6 & 74.2 \\
Ferritin, ng/ml & 397 & 83.3 & 93.1 & 93.2 & 83.1 & 0.78 \\
CRP, mg/l & 54 & 63.6 & 79.3 & 77.8 & 65.7 & 71 \\
SAA + ferritin & & 100 & 100 & 100 & 100 & 0.86 \\
\hline
\end{tabular}

SAA, serum amyloid A; CEA, carcinoembryonic antigen; CRP, C-reactive protein.

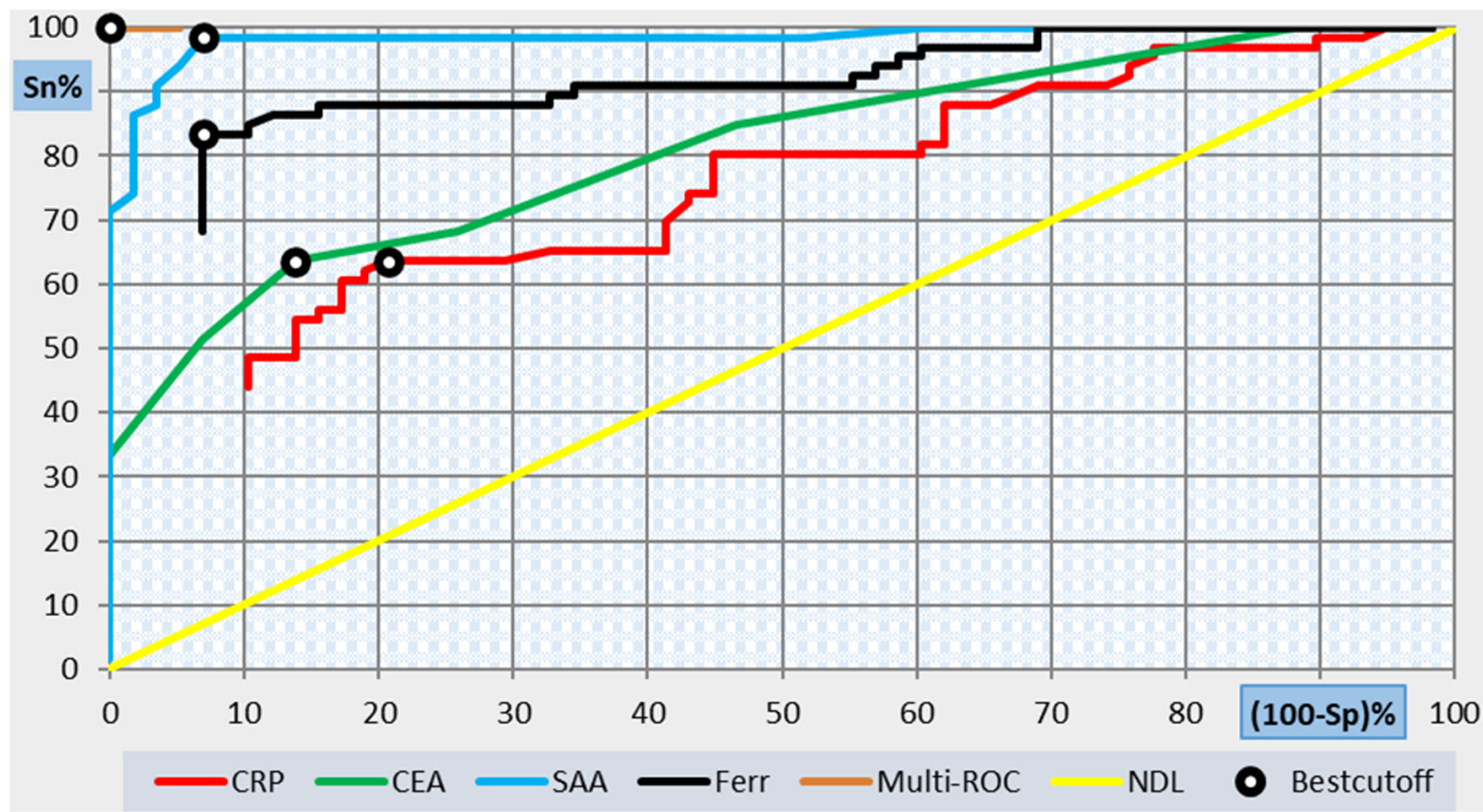

Figure 1. Individual and multi-ROC curves showing the performance of all studied parameters in predicting severity in COVID-19 patients. SP, specificity; Sn, Sensitivity; CRP, C-reactive protein; CEA, carcinoembryonic antigen; SAA, serum amyloid A; Ferr, ferritin; ROC, receiver operating characteristic; NDL: Non-Diagnostic Line.

women (31). In the present study, several social, cultural and behavioral differences between the sexes may have contributed to the high overall male to female ratio ( 79 to $21 \%$, respectively) seen in this cohort, including habits more common in men, such as smoking $(32,33)$ and sex-based differences in hygiene. Additionally, based on anecdotal evidence, men are likely to leave their houses and enter crowded areas in Egypt. Furthermore, differences in health-seeking behaviors, unequal access to healthcare facilities and testing between sexes may have skewed the data further towards a male bias $(34,35)$.

However, a consistent feature of the COVID-19 pandemic is the male bias towards severe disease (36). This agrees with the results of the present study, as $85 \%$ (56 out of 66) of the severe cases were men compared with $15 \%$ of women. Male sex is associated with nearly a $3 x$ risk of requiring intensive care unit admission and a higher probability of death compared to women (31).
Fundamental differences in the immune response between males and females are likely to be the driving factor behind the significant sex bias observed in severe COVID-19 cases. Sex differences in innate and adaptive immunity have been reported, which may account for the reduced risk of severe disease in females. A robust antiviral innate IFN response and enhanced adaptive immunity in females, higher numbers of $\mathrm{CD}^{+} \mathrm{T}$ cells, robust $\mathrm{CD}^{+} \mathrm{T}$ cells and increased $\mathrm{B}$ cell production of immunoglobulin compared to males, may lead to more effective viral control in females, and a relatively lower risk of developing severe disease $(37,38)$. The $\mathrm{X}$ chromosome encodes several immune-related genes, which can be variably expressed on both alleles, increasing the diversity of the immune response (39). Hormonal differences also play a role; estradiol augments the immune response in contrast to testosterone, which was found to suppress the immune system (40). 
The median WBC, PNL and PLT counts were higher in the severe group than in the mild group, whereas the lymphocyte count was lower. However, the differences between both groups regarding these parameters were not statistically significant. Of the 124 COVID-19 positive cases, a high prevalence of increased inflammatory marker levels was identified. These results showed that in all COVID-19 cases, there was an overall increased level of inflammatory parameters, including SAA, CEA, CRP and ferritin, in addition to pro-coagulation markers. D-Dimer levels were similar to the results of previous studies $(29,41,42)$, and they were significantly higher at admission in the severe patient group than in the mild group, whereas total WBC, lymphocyte and PLT counts were within the normal range, consistent with the results of Li et al (42) and Wang et al (43).

Huang et al (44) showed that patients with severe COVID-19 had higher levels of IL-1 $\beta$, IFN- $\gamma$, IP-10 and monocyte chemoattractant protein compared with mild cases, causing Th1 cell activation and stimulating the production of SAA, CRP, procalcitonin and PLT. These inflammatory factors may be useful as indicators reflecting the body's response to infection (45).

According to Li et al (42) and Wang et al (43) as the disease progressed from mild to severe, SAA and CRP levels increased, while lymphocyte counts gradually decreased. However, WBC and PLT were all within normal ranges, suggesting that SAA and CRP are closely related to disease classification, while WBC and PLT are of little significance.

SAA showed a highly significant positive correlation with age, disease duration, neutrophil count and all inflammatory indices, including CRP, ferritin, CEA and D-Dimer in the present study. Similarly, CEA showed a significant positive correlation with age, disease duration and all inflammatory indices. Additionally, both SAA and CEA levels showed a highly significant positive correlation with the CT-SS. These results agreed with the results from various studies showing significantly higher SAA levels in PCR positive COVID-19 cases, and significant associations between SAA levels and the number of COVID-19 cases, severity and mortality rate $(29,41,42)$.

SAA is an acute phase protein produced by the liver and is induced by cytokines, including IL-1 $\beta$, IL- 6 and TNF- $\alpha$. SAA promotes an inflammatory response by activating chemokines and inducing chemotaxis, even at very low concentrations $(46,47)$. In current clinical practices, SAA is frequently used as an indicator for monitoring of inflammation and estimation of prognosis $(48,49)$. SAA showed promising results when used to monitor the effectiveness of antibiotics (cefotiam and Augmentin) in early onset neonatal sepsis according to a study by Liu et al (49).

Previous studies have shown that patients with severe ARDS have significantly increased levels of SAA, suggesting that SAA could be used as a biomarker to monitor the progression of respiratory diseases $(50,51)$. As stated by Cheng et al (52), in a study involving 89 COVID-19 patients, dynamic changes in SAA could be used to predict prognosis.

In the present study, serum CEA levels were significantly higher in patients with severe COVID-19 than in those with a mild infection. Moreover, higher serum CEA levels were associated with a higher CT-SS. A limited number of studies reported elevated serum CEA levels in COVID-19 patients with significantly increased CEA levels compared with healthy controls, and a potential association between CEA and CRP levels was reported $(53,54)$. However, its role in predicting clinical outcomes or CT involvement in patients with COVID-19 is still under investigation. The present study validated the previous results that CEA levels were related to COVID-19 severity. Moreover, the association between CEA levels and CT scores were also validated, which are in agreement with the findings of Chen et al (41).

CEA is a biomarker of adenocarcinoma in respiratory or digestive system cancers, in addition to non-neoplastic lung diseases $(55,56)$. Increased CEA expression was detected in type II pneumocytes in allergic bronchopulmonary aspergillosis (57) and atypical epithelial proliferation in idiopathic pulmonary fibrosis $(13,58)$. Bronchiolar and type II alveolar epithelial cells are the main targets of SARS-CoV-2 in the lungs. SARS-CoV-2 infection-induces massive type II alveolar epithelial cell death and aberrant regeneration of type II pneumocytes along with the production of CEA, such as that observed in the uncontrolled proliferation in lung adenocarcinoma. Moreover, atypical epithelial and fibroblast proliferation may also worsen the obstruction of bronchioles and lung consolidation, causing refractory hypoxemia along with worse CT scores $(59,60)$.

Consequently, it is possible that serum CEA levels may correlate with the severity and prognosis of COVID-19. Based on the relationship between CEA and type II pneumocyte hyperplasia and lung fibrosis, medications such as nintedanib, which target atypical epithelial and fibrotic proliferation, may be a potential therapeutic option to decrease mortality in patients with COVID-19 (41).

An elevated D-Dimer level represents microangiopathy and a hypercoagulable state in COVID-19 patients (61). COVID-19 is implicated in aggressive pro-inflammatory responses causing endothelial cell dysfunction and excessive thrombin formation (62), which can be responsible for oxygen desaturation and respiratory distress seen in severe cases $(63,64)$. In the present study, D-Dimer levels were significantly higher in patients with severe COVID-19, and there was a highly significant positive correlation with SAA and CEA in addition to CRP and ferritin levels. These results are also in agreement with a retrospective study of 183 patients with COVID-19 performed by Tang et al (65) which showed a significant increase in D-Dimer levels and fibrin degradation products, and this was indicative of a poor prognosis.

Similarly, CRP is also an acute phase protein, the levels of which rise rapidly, and the rate of increase is positively correlated with the severity of infection $(7,29)$. A higher CRP level is linked to an increased risk of severe COVID-19 and may contribute to pneumonia, ARDS and the rapid multiple organ damage $(7,66)$. According to a study by Liu et al $(66)$, elevated CRP and decreased albumin levels were important factors affecting the prognosis of COVID-19.

Ferritin is another crucial mediator of the immune response that showed a statistically significant increase in severe COVID-19 cases, and showed a highly significant positive correlation with SAA levels in addition to CEA, CRP and D-Dimer levels in the present study. Increased ferritin levels may be implicated in the cytokine storm due to its 
direct immunosuppressive and pro-inflammatory effects (25). Efstathiou et al (67) indicated that viral infections increased ferritin levels. This finding may be attributed to the fact that the inflammatory mediators induce an increase in ferritin levels, in addition to denaturation and necrosis of cells to break down cell membranes, causing a leakage of ferritin from damaged cells (68).

The results of the present study showed that the levels of SAA, CEA, CRP and ferritin increased as the disease severity increased. CT imaging is an important clinical diagnostic tool for evaluating COVID-19 infections. According to the correlation analysis in this study, SAA, CEA, CRP and ferritin levels at admission were highly correlated with the CT-SS, suggesting a possible role in predicting disease progression.

ROC curve analysis was used to comprehensively and accurately compare and evaluate the diagnostic performance of SAA, CEA, CRP and ferritin levels on admission and to explore their clinical and prognostic utility. The results of the current study showed that the AUC was highest for SAA, followed by ferritin, CEA and CRP. According to the multivariate regression analysis and ROC curve results, the combined use of SAA and ferritin was more sensitive than SAA or ferritin alone as predictors of severity, as their combination had the highest predictive value for disease severity, with an AUC of 1.000 .

Accordingly, the combined detection of SAA and ferritin may have guiding significance for assessing the severity, disease progression and prognosis of COVID-19 cases. This may aid effective intervention measures to be implemented in timely manner and reduce the rates of severe illness and mortality, as we continue to face upcoming waves of COVID-19.

The present study has some limitations. The elevations in SAA and CEA may have been due to various other conditions and comorbidities, which themselves may be associated with a higher COVID-19 risk. Additionally, this study was a single-center study with a relatively small cohort, which may have limited the power of the statistical analyses.

In conclusion, the combined detection of SAA and ferritin may have guiding significance for the severity of COVID-19 and may be correlated with CT-SS in patients with COVID-19. Serum CEA levels were correlated with the severity of the CT scores and the prognosis of COVID-19. Therefore, assessment and monitoring of these laboratory markers at the earliest stage of the disease may have a significant impact on halting disease progression and decreasing mortality. Further prospective and multicenter studies with validation cohorts should be performed in the future.

\section{Acknowledgements}

Not applicable.

\section{Funding}

No funding was received.

\section{Availability of data and materials}

The datasets used and/or analyzed during the present study are available from the corresponding author on reasonable request.

\section{Authors' contributions}

DAA and MAEMT designed the study, contributed to data collection and interpretation, and wrote the manuscript. NMBE assisted with sample collection, data interpretation and editing of the manuscript. FMB and MAEM contributed to sample collection, data interpretation and drafting of the manuscript. FMB and MAEMT confirm the authenticity of all the raw data. All authors have read and approved the final manuscript.

\section{Ethics approval and consent to participate}

This study was conducted in accordance with the principles outlined in the Declaration of Helsinki of the World Medical Association. The study was approved by the Institutional Ethics Committee of Ain Shams University. Informed consent was obtained from all enrolled participants after receiving an explanation of the study's aim and procedures.

\section{Patient consent for publication}

Not applicable.

\section{Competing interests}

The authors declare that they have no competing interests.

\section{References}

1. Gorbalenya AE, Baker SC, Baric RS, De Groot RJ, Drosten C, Gulyaeva AA, Haagmans BL, Lauber C, Leontovich AM, et al; Coronaviridae Study Group of the International Committee on Taxonomy of Viruses: The species Severe acute respiratory syndrome-related coronavirus: Classifying 2019-nCoV and naming it SARS-CoV-2. Nat Microbiol 5: 536-544, 2020.

2. Zhou F, Yu T, Du R, Fan G, Liu Y, Liu Z, Xiang J, Wang Y, Song B, Gu X, et al: Clinical course and risk factors for mortality of adult inpatients with COVID-19 in Wuhan, China: A retrospective cohort study. Lancet 395: 1054-1062, 2020.

3. Calina D, Docea AO, Petrakis D, Egorov AM, Ishmukhametov AA, Gabibov AG, Shtilman MI,Kostoff R, Carvalho F, Vinceti M, et al: Towards effective COVID-19 vaccines: Updates, perspectives and challenges (Review). Int J Mol Med 46: 3-16, 2020.

4. Khan M, Khan H, Khan S and Nawaz M: Epidemiological and clinical characteristics of coronavirus disease (COVID-19) cases at a screening clinic during the early outbreak period: A single-centre study. J Med Microbiol 69: 1114-1123.2020.

5. Lu R, Zhao X, Li J, Niu P, Yang B, Wu H, Wang W, Song H, Huang B, Zhu N, et al: Genomic characterisation and epidemiology of 2019 novel coronavirus: Implications for virus origins and receptor binding. Lancet 395: 565-574, 2020.

6. Ye Q, Wang B and Mao J: The pathogenesis and treatment of the 'Cytokine Storm' in COVID-19. J Infect 80: 607-613, 2020.

7. Tan C, Huang Y, Shi F, Tan K, Ma Q, Chen Y, Jiang X and Li X: C-reactive protein correlates with computed tomographic findings and predicts severe COVID-19 early. J Med Virol 92: 856-862, 2020.

8. Wilson PG, Thompson JC, Webb NR, de Beer FC, King VL and Tannock LR: Serum amyloid A, but not C-reactive protein, stimulates vascular proteoglycan synthesis in a pro-atherogenic manner. Am J Pathol 173: 1902-1910, 2008.

9. Vietri L, Fui A, Bergantini L, d'Alessandro M, Cameli P, Sestini P, Rottoli P and Bargagli E: Serum amyloid A: A potential biomarker of lung disorders. Respir Investig 58: 21-27, 2020.

10. Goldenberg DM, Neville AM, Carter AC, Go VL, Holyoke ED, Isselbacher KJ, Schein PS and Schwartz M: CEA (carcinoembryonic antigen): Its role as a marker in the management of cancer. J Cancer Res Clin Oncol 101: 239-242, 1981. 
11. Hao C, Zhang G and Zhang L: Serum CEA levels in 49 different types of cancer and noncancer diseases. Prog Mol Biol Transl Sci 162: 213-227, 2019.

12. Liu Q, Wang RS, Qu GQ, Wang YY, Liu P, Zhu YZ, Fei G, Ren L, Zhou YW and Liu L: Gross examination report of a COVID-19 death autopsy. Fa Yi Xue Za Zhi 36: 21-23, 2020 (In English, Chinese).

13. Fahim A, Crooks MG, Wilmot R, Campbell AP, Morice AH and Hart SP: Serum carcinoembryonic antigen correlates with severity of idiopathic pulmonary fibrosis. Respirology 17: 1247-1252, 2012.

14. WHO: World Health Organization. Coronavirus Disease (COVID-19) Outbreak. World Health Organization, Geneva, 2020

15. Teama MA, Abdelhakam DA, Elmohamadi MA and Badr FM: Vitamin D deficiency as a predictor of severity in patients with COVID-19 infection. Sci Prog 104: 368504211036854, 2021.

16. World Medical Association: World Medical Association Declaration of Helsinki: Ethical principles for medical research involving human subjects. JAMA 310: 2191-2194, 2013.

17. Prokop M, van Everdingen W, van Rees Vellinga T, Quarles van Ufford H, Stöger L, Beenen L, Geurts B, Gietema H, Krdzalic J, Schaefer-Prokop C, et al; COVID-19 Standardized Reporting Working Group of the Dutch Radiological Society: CO-RADS A categorical CT assessment scheme for patients suspected of having COVID-19-definition and evaluation. Radiology 296: E97-E104, 2020

18. Francone M, Iafrate F, Masci GM, Coco S, Cilia F, Manganaro L, Panebianco V, Andreoli C, Colaiacomo MC,Zingaropoli MA, et al Chest CT score in COVID-19 patients: Correlation with disease severity and short-term prognosis. Eur Radiol 30: 6808-6817, 2020

19. Zhu N, Zhang D, Wang W, Li X, Yang B, Song J, Zhao X, Huang B, Shi W, et al: A novel coronavirus from patients with pneumonia in China. N Engl J Med 382: 727-733, 2020

20. Adhikari SP, Meng S, Wu YJ, Mao YP, Ye RX, Wang QZ, Sun C, Sylvia S, Rozelle S, Raat H, et al: Epidemiology, causes, clinical manifestation and diagnosis, prevention and control of coronavirus disease (COVID-19) during the early outbreak period: A scoping review. Infect Dis Poverty 9: 29. 2020.

21. Xu XW, Wu XX, Jiang XG, Xu KJ, Ying LJ, Ma CL, Li SB, Wang HY, Zhang S, Gao HN, et al: Clinical findings in a group of patients infected with the 2019 novel coronavirus (SARS-Cov2) outside of Wuhan, China: Retrospective case series. BMJ: Feb 19, 2020 (Epub ahead of print).

22. Guan W, Ni Z, Hu Y, Liang W, Ou C, He J, Liu L, Shan H, Lei C, Hui DS, et al: Clinical characteristics of coronavirus disease 2019 in China. N Engl J Med 382: 1708-1720, 2020.

23. Wu J, Liu J, Li S, Peng Z, Xiao Z, Wang X, Yan R and Luo J: Detection and analysis of nucleic acid in various biological samples of COVID-19 patients. Travel Med Infect Dis 37: 101673, 2020.

24. Lin C, Ding Y, Xie B, Sun Z, Li X, Chen Z and Niu M: Asymptomatic novel coronavirus pneumonia patient outside Wuhan: The value of CT images in the course of the disease. Clin Imaging 63: 7-9, 2020.

25. Vargas-Vargas $M$ and Cortés-Rojo C: Ferritin levels and COVID-19. Rev Panam Salud Publica 44: e72, 2020.

26. Yang Z, Shi J, He Z, Lu Y, Xu Q, Ye C, Chen S, Tang B, Yin K, $\mathrm{Lu} \mathrm{Y}$, et al: Predictors for imaging progression on chest CT from coronavirus disease 2019 (covid-19) patients. Aging (Albany NY) 12: 6037-6048.2020.

27. Zheng F, Tang W, Li H, Huang YX, Xie YL and Zhou ZG: Clinical characteristics of 161 cases of corona virus disease 2019 (COVID-19) in Changsha. Eur Rev Med Pharmacol Sci 24 3404-3410, 2020.

28. Ishiguro T, Kagiyama N, Uozumi R, Odashima K, Takaku Y, Kurashima K, Morita S and Takayanagi N: Clinical characteristics of influenza-associated pneumonia of adults: Clinical features and factors contributing to severity and mortality. Yale J Biol Med 90: 165-181, 2017.

29. Liu SL, Wang SY, Sun YF, Jia QY, Yang CL, Cai PJ, Li JY, Wang L and Chen Y: Expressions of SAA, CRP, and FERR in different severities of COVID-19. Eur Rev Med Pharmacol Sci 24: 11386-11394, 2020.

30. CAPMAS, Central Agency for Public Mobilization and Statistics: 2021. Available from: https://www.capmas.gov.eg/.

31. Peckham H, De Gruijter NM, Raine C, Radziszewska A, Ciurtin C, Wedderburn LR, Rosser EC, Webb K and Deakin CT: Male sex identified by global COVID-19 meta-analysis as a risk factor for death and ITU admission. Nat Commun 9: 11: 6317.2020
32. Giovino GA, Mirza SA, Samet JM, Gupta PC, Jarvis MJ, Bhala N, Peto R, Zatonski W, Hsia J, Morton J, et al: Tobacco use in 3 billion individuals from 16 countries: An analysis of nationally representative cross-sectional household surveys. Lancet 380: 668-679, 2012

33. Loffredo CA, Radwan GN, Eltahlawy EM, El-Setouhy M, Magder L and Hussein MH: Estimates of the prevalence of tobacco smoking in Egypt. Open J Epidemiol 5: 129-135, 2015.

34. Spagnolo PA, Manson JAE and Joffe H: Sex and gender differences in health: What the COVID-19 pandemic can teach us. Ann Intern Med 173: 385-386, 2020

35. Regitz-Zagrosek V: Sex and gender differences in health. Science and society series on sex and science. EMBO Rep 13: 596-603, 2012.

36. Singh S, Chowdhry M, Chatterjee A and Khan A: Gender-based disparities in COVID-19: Clinical characteristics and propensity matched analysis of outcomes. MedRxiv: May 12, 2020 (Epub ahead of print).

37. Abdullah M, Chai PS, Chong MY, Tohit ERM, Ramasamy R, Pei CP and Vidyadaran S: Gender effect on in vitro lymphocyte subset levels of healthy individuals. Cell Immunol 272: 214-219, 2012.

38. Hewagama A, Patel D, Yarlagadda S, Strickland FM and Richardson BC: Stronger inflammatory/cytotoxic T-cell response in women identified by microarray analysis. Genes Immun 10: 509-516, 2009.

39. Gal-Oz ST, Maier B, Yoshida H, Seddu K, Elbaz N, Czysz C, Zuk O, Stranger BE, Ner-Gaon H and Shay T: ImmGen report: Sexual dimorphism in the immune system transcriptome. Nat Commun 10: 4295, 2019.

40. Klein SL and Flanagan KL: Sex differences in immune responses. Nat Rev Immunol 16: 626-638, 2016.

41. Chen Q, Kong H, Qi X, Ding W, Ji N, Wu C, Huang C, Wu W, Huang M, Wu W, et al: Carcinoembryonic antigen: A potential biomarker to evaluate the severity and prognosis of COVID-19. Front Med (Lausanne) 7: 579543, 2020.

42. Li H, Xiang X, Ren H, Xu L, Zhao L, Chen X, Long H, Wang Q and $\mathrm{Wu} \mathrm{Q}$ : Serum amyloid A is a biomarker of severe coronavirus disease and poor prognosis. J Infect 80: 646-655, 2020.

43. Wang D, Hu B, Hu C, Zhu F, Liu X, Zhang J, Wang B, Xiang H, Cheng Z, Xiong Y, et al: Clinical characteristics of 138 hospitalized patients with 2019 novel coronavirus-infected pneumonia in Wuhan, China. JAMA 323: 1061-1069, 2020.

44. Huang C, Wang Y, Li X, Ren L, Zhao J, Hu Y, Zhang L, Fan G, Xu J, Gu X, et al: Clinical features of patients infected with 2019 novel coronavirus in Wuhan, China. Lancet 395: 497-506, 2020.

45. Behrens K and Alexander WS: Cytokine control of megakaryopoiesis. Growth Factors 36: 89-103, 2018.

46. Connolly M, Rooney PR, Mcgarry T, Maratha AX, Mccormick J, Miggin SM, Veale DJ and Fearon U: Acute serum amyloid A is an endogenous TLR2 ligand that mediates inflammatory and angiogenic mechanisms. Ann Rheum Dis 75: 1392-1398, 2016.

47. Sack GH: Serum amyloid A - A review. Mol Med 24: 46, 2018.

48. Wakai M, Hayashi R, Tanaka S, Naito T, Kumada J, Nomura M, Takigawa $\mathrm{H}$, Oka S, Ueno Y, Ito M, et al: Serum amyloid A is a better predictive biomarker of mucosal healing than C-reactive protein in ulcerative colitis in clinical remission. BMC Gastroenterol 20: 85, 2020.

49. Liu C, Zhang Y, Shang Y, Fang C, He Q and Xie L: Clinical values of common biomarkers for efficacy monitoring of antibiotics in early onset neonatal sepsis. Transl Pediatr 9: 669-676, 2020.

50. Yip TT, Chan JW, Cho WC, Yip TT, Wang Z, Kwan TL, Law SC, Tsang D, Chan JK, Lee KC, et al: Protein chip array profiling analysis in patients with severe acute respiratory syndrome identified serum amyloid A protein as a biomarker potentially useful in monitoring the extent of pneumonia. Clin Chem 51: 47-55, 2005.

51. Zinellu A, Paliogiannis P, Carru C and Mangoni AA: Serum amyloid A concentrations, COVID-19 severity and mortality: An updated systematic review and meta-analysis. Int J Infect Dis 105: 668-674, 2021.

52. Cheng L, Yang JZ, Bai WH, Li ZY, Sun LF, Yan JJ, Zhou CL and Tang BP: Prognostic value of serum amyloid A in patients with COVID-19. Infection 48: 715-722, 2020.

53. Wei X, Su J, Yang K, Wei J, Wan H, Cao X, Tan W and Wang H: Elevations of serum cancer biomarkers correlate with severity of COVID-19. J Med Virol 92: 2036-2041, 2020.

54. Yang C, Wang J, Liu J, Huang S and Xiong B: Elevated carcinoembryonic antigen in patients with COVID-19 pneumonia. J Cancer Res Clin Oncol 146: 3385-3388, 2020. 
55. He G, Jiang Z, Xue S, Sun X and Wang W: Expression of LDH and CEA in serum in the process of targeted therapy of lung adenocarcinoma and the association between them and prognosis. Oncol Lett 17: 4550-4556, 2019.

56. Mohr AM, Gould JJ, Kubik JL, Talmon GA, Casey CA, Thomas P, Tuma DJ and McVicker BL: Enhanced colorectal cancer metastases in the alcohol-injured liver. Clin Exp Metastasis 34: 171-184, 2017.

57. Noguchi T, Yamamoto K, Moriyama G, Saito Y, Kyoyama H, Mikami S, Ono R, Kobayashi T, Yamana K and Uematsu K: Evaluation of serum levels of carcinoembryonic antigen in allergic bronchopulmonary aspergillosis. J Nippon Med School 80: 404-409, 2013.

58. Abbona GC, Papotti M, Gugliotta P, Pecchio F and Rapellino M: Immunohistochemical detection of carcinoembryonic antigen (CEA) in non-neoplastic lung disease. Int J Biol Markers 8: 240-243, 1993.

59. Yao XH, Li TY, He ZC, Ping YF, Liu HW, Yu SC, Mou HM, Wang LH, Zhang HR, et al: A pathological report of three COVID-19 cases by minimally invasive autopsies. Zhonghua Bing Li Xue Za Zhi: 49: 411-417, 2020 (In Chinese).

60. Fox SE, Akmatbekov A, Harbert JL, Li G, Brown JQ and Heide RS: Pulmonary and cardiac pathology in African American patients with COVID-19: An autopsy series from New Orleans. Lancet Respir Med 8: 681-686, 2020.

61. Zhang L, Yan X, Fan Q, Liu H, Liu X, Liu Z and Zhang Z: D-Dimer levels on admission to predict in-hospital mortality in patients with Covid-19. J Thromb Haemost 18: 1324-1329, 2020.

62. Levi M and Van der Poll T: Coagulation and sepsis. Thromb Res 149: 38-44, 2017.
63. Gattinoni L, Coppola S, Cressoni M, Busana M, Rossi S and Chiumello D: COVID-19 does not lead to a 'typical' acute respiratory distress syndrome. Am J Respir Crit Care Med 201: 1299-1300, 2020

64. Oudkerk M, Buller HR, Kuijpers D, van Es N, Oudkerk SF, McLoud T, Gommers D, Dissel JV, Cate HT and van Beek EJ: Diagnosis, prevention, and treatment of thromboembolic complications in COVID-19: Report of the national institute for public health of the Netherlands. Radiology 297: E216-E222, 2020.

65. Tang N, Li D, Wang X and Sun Z: Abnormal coagulation parameters are associated with poor prognosis in patients with novel coronavirus pneumonia. J Thromb Haemost 18: 844-847, 2020.

66. Liu W, Tao ZW, Wang L, Yuan ML, Liu K, Zhou L, Wei S, Deng Y, Liu J, Liu HG, et al: Analysis of factors associated with disease outcomes in hospitalized patients with 2019 novel coronavirus disease. Chin Med J (Engl) 133: 1032-1038, 2020.

67. Efstathiou SP, Pefanis AV, Tsiakou AG, Skeva II, Tsioulos DI, Achimastos AD and Mountokalakis TD: Fever of unknown origin: Discrimination between infectious and non-infectious causes. Eur J Intern Med 21: 137-143, 2010.

68. Kell DB and Pretorius E: Serum ferritin is an important inflammatory disease marker, as it is mainly a leakage product from damaged cells. Metallomics 6: 748-773, 2014.

This work is licensed under a Creative Commons Attribution-NonCommercial-NoDerivatives 4.0 International (CC BY-NC-ND 4.0) License. 